\title{
The Clinical COPD Questionnaire: response to pulmonary rehabilitation and minimal clinically important difference
}

\author{
Samantha S C Kon, ${ }^{1}$ Deniz Dilaver, ${ }^{2}$ Manvi Mittal, ${ }^{1}$ Claire M Nolan, ${ }^{1,2}$ Amy L Clark, ${ }^{2}$ \\ Jane L Canavan, ${ }^{1}$ Sarah E Jones, ${ }^{1}$ Michael I Polkey, ${ }^{1}$ William D-C Man ${ }^{1,2}$
}

${ }^{1}$ NIHR Respiratory Biomedical Research Unit, Royal Brompton \& Harefield NHS Foundation Trust and Imperial College, Harefield, UK

${ }^{2}$ Harefield Pulmonary

Rehabilitation Unit, Royal Brompton \& Harefield NHS Foundation Trust, Harefield, UK

\section{Correspondence to} Dr Samantha Kon, Department of Respiratory Medicine, Harefield Hospital, Hill End Road, Harefield UB9 6JH, UK; s.kon@rbht.nhs.uk

Received 2 July 2013 Revised 4 September 2013 Accepted 3 October 2013 Published Online First 22 October 2013
CrossMark

To cite: Kon SSC, Dilaver D, Mittal M, et al. Thorax 2014;69:793-798.

\section{ABSTRACT}

Background The Clinical COPD Questionnaire (CCQ) is a simple 10-item, health-related quality of life questionnaire (HRQoL) with good psychometric properties. However, little data exists regarding the responsiveness of the CCQ to pulmonary rehabilitation (PR) or the minimal clinically important difference (MCID). The study aims were to assess the responsiveness of the $C C Q$ to $P R$, to compare the responsiveness of the CCQ to other HRQoL questionnaires and to provide estimates for the MCID. Methods The CCQ, St George's Respiratory Questionnaire (SGRQ), Chronic Respiratory Questionnaire (CRQ) and COPD Assessment Test (CAT) were measured in 261 patients with COPD before and after outpatient PR. Pre to post PR changes and Cohen's effect size were calculated. Changes in CCQ were compared with changes in other HRQoL questionnaires. Using an anchor-based approach and receiver operating characteristic (ROC) curves, the CCQ change cutoffs that identified patients achieving the known MCID for other health status questionnaires with PR were identified. Results The CCQ, SGRQ, CRQ and CAT all significantly improved with PR with an effect size of $-0.39,-0.33$, 0.62 and -0.25 , respectively. CCQ change correlated significantly with change in SGRQ, CRQ and CAT $(r=0.48,-0.56,0.54$, respectively; all $p<0.001)$. ROC curves consistently identified a CCQ change cutoff of -0.4 as the best discriminating value to identify the MCID for the SGRQ, CRQ and CAT (area under curve: $0.71,0.75$ and 0.77 , respectively; all $p<0.001$ ).

Conclusions The CCQ is responsive to PR with an estimated clinically important improvement of -0.4 points. The CCQ is a practical alternative to more timeconsuming measures of HRQoL.

\section{INTRODUCTION}

Health-related quality of life (HRQoL) is an important outcome measure in patients with COPD, and increasingly used to quantify symptom burden and guide treatment. ${ }^{1}$ Although there are numerous questionnaires designed to measure HRQoL in COPD, the most widely used questionnaires are the $\mathrm{St}$ George's Respiratory Questionnaire (SGRQ) ${ }^{2}$ and the Chronic Respiratory Questionnaire (CRQ), ${ }^{3}$ which are well validated, reliable and responsive to change. However, these questionnaires may be time consuming to complete, need health professional input, require specialist software to use, or are complex to score.

\section{Key messages}

What is the key question?

- Does the Clinical COPD Questionnaire (CCQ), a simple, easy-to-complete health status questionnaire, show a response to pulmonary rehabilitation?

What is the bottom line?

- The CCQ decreases (improves) with pulmonary rehabilitation, with a clinically important improvement estimated as a fall of 0.4 points. Change in CCQ with pulmonary rehabilitation correlates significantly with change in other well established disease-specific questionnaires.

\section{Why read on?}

- This study provides evidence that the CCQ is as responsive as other well established COPD-specific health status instruments, and is a practical alternative compared with more time-consuming questionnaires.

Recently, two shorter and simpler HRQoL questionnaires for patients with COPD have been described and validated. The COPD Assessment Test (CAT) consists of eight items, each scored between 0 and 5 , providing a total score out of $40 .{ }^{4}$ It is self-completed and is easily scored by adding the scores of the eight individual items, with higher scores representing greater negative impact of disease on HRQoL. The Clinical COPD Questionnaire (CCQ) consists of 10 items (each scored between 0 and 6), divided into three domains (symptoms, functional, mental). ${ }^{5}$ The total score is calculated by summing the scores of the individual items and dividing by 10 (the number of individual items) giving a total score between 0 and 6 with higher scores representing worse HRQoL.

The CAT and CCQ show good psychometric properties and take less than 2 min to complete. Recently, the Global Initiative for Chronic Obstructive Lung Disease (GOLD) guidelines recommended the use of the CAT questionnaire to assess symptom burden in patients with COPD and assist in guiding management. ${ }^{1}$ 
Despite being described 6 years earlier, the CCQ is arguably less well known than the CAT. However, there is indirect evidence that the CCQ may have some advantages over the CAT. Patients with COPD participating in pulmonary rehabilitation (PR) show a greater need for assistance to complete the CAT compared with the CCQ. ${ }^{6}$ Similarly, in a study comparing the validity of the CAT, CCQ and SGRQ, more than $60 \%$ of patients reported the CCQ being a better tool than the CAT for assessing health status. ${ }^{7}$ Furthermore, in a multicentre study designed to assess the responsiveness of the CAT to PR, effect size for the CCQ was observed to be higher than the CAT $(0.6$ vs 0.4 ), although this was in a small subset of the study cohort. ${ }^{8}$ Recently the International Primary Care Respiratory Group reviewed nine COPD 'Wellness' tools, including the CCQ, CAT, CRQ and SGRQ, and concluded that the CCQ was the most suitable tool for use in primary care. ${ }^{9}$

$\mathrm{PR}$ is the cornerstone of management in COPD. Recently there have been several publications reporting the responsiveness of the CAT to PR. ${ }^{8} 10{ }^{11}$ However, little data exist regarding the responsiveness of the CCQ to PR. Only one previous study has estimated the minimal clinically important difference (MCID) for the CCQ using anchor-based and distribution-based approaches. ${ }^{12}$ Therefore, the aims of the study were to assess the responsiveness of the CCQ to PR, compare the responsiveness of the CCQ to other well established HRQoL questionnaires and to provide further data to corroborate previous estimates of the MCID for the CCQ. We hypothesised that the CCQ would decrease significantly with PR, and would be similarly responsive to the effects of PR as the CAT.

\section{METHODS}

\section{Participants}

Participants were recruited from Harefield Hospital between November 2011 and January 2013. Inclusion criteria included a diagnosis of COPD, according to GOLD criteria, ${ }^{13}$ clinical indication for PR according to British Thoracic Society guidelines ${ }^{14}$ and no exacerbation in the past 6 weeks. Exclusion criteria included any condition that might make exercise unsafe (eg, unstable cardiac disease, inability to walk $5 \mathrm{~m}$ without assistance), predominant neurological limitation to walking (eg, significant hemiplegia) or previous PR within the past 12 months. All participants gave informed consent and the study was approved by the West London and the London-Camberwell St Giles Research Ethics Committees.

\section{Measurements and PR}

The weekly version of the CCQ was prospectively measured before and after PR. Other outcome variables included the incremental shuttle walk (ISW), ${ }^{15}$ the SGRQ, ${ }^{2}$ the self-reported $\mathrm{CRQ}^{3}$ and the CAT. Patients were kept blinded to their performance in their questionnaires and walking tests until the completion of all study assessments. PR comprised an 8-week outpatient-based programme, consisting of two supervised exercise and education sessions per week. Patients were given a choice of four hospital and community-based locations around northwest London but classes were run by the same team with the same equipment. Each supervised session lasted $2 \mathrm{~h}$, comprising $1 \mathrm{~h}$ of exercise (aerobic walking and cycling, strength training for the upper and lower limb), and $1 \mathrm{~h}$ of educational activities (with an emphasis on self-management) delivered by a multidisciplinary team. Aerobic exercise intensity was initially set at $60-80 \%$ of the predicted $\mathrm{VO}_{2}$ max and increased according to dyspnoea, as measured using the modified Borg breathlessness scale. Patients also received an individualised home exercise programme and diary, which encouraged at least 20 min of exercise per day.

\section{Sample size}

We aimed to study a minimum of 250 PR COPD completers, in line with previous reports in this area. ${ }^{8}$ From previous audits of the Harefield PR service, the dropout from PR is approximately $25 \%$. We therefore aimed to recruit a minimum of 333 patients over the time period.

\section{Data analysis}

Data analyses and graphical presentations were performed using GraphPad Prism 5 (GraphPad Software, San Diego, California, USA) or SPSS version 21 (IBM, Armonk, New York, USA). Only data from participants who attended pre and post PR assessments and completed the CCQ were analysed. Paired $t$ tests were used to compare outcomes before and after PR. Pearson correlation was used to assess the relationship between change in CCQ and change in other health status instruments (SGRQ, CRQ and CAT). Analysis of variance (ANOVA) was used to compare multiple groups. Cohen's d effect size was calculated as the mean difference in outcome variable before and after PR divided by the mean SD (ie, mean SD at baseline and following PR).

\section{Determination of MCID}

To estimate the MCID, the change in CCQ with PR was anchored against change in the other HRQoL questionnaires (SGRQ, CRQ and CAT) as these measure a similar construct to the CCQ. The mean (95\% CI) CCQ changes according to whether patients achieved or failed to achieve the accepted MCID for the SGRQ total score (decline of 4 points or more) ${ }^{16}$ and for the CRQ total score (an increase in 10 points or more $)^{17}$ with PR were calculated. The MCID for the CAT has not been formally established, but based on the relationship with SGRQ, a decrease of 4 points in SGRQ is equivalent to a decrease of 1.6 points in the CAT. ${ }^{4}$ As the CAT score is expressed as an integer on an individual level, we therefore calculated the mean (95\% CI) change in CCQ according to whether patients showed at least a 2-point reduction in CAT or not with PR. To identify the MCID for the CCQ, we plotted receiver operating characteristic (ROC) curves to determine the change in CCQ with equal sensitivity and specificity to discriminate between 'improved' (those achieving the MCID for the HRQoL questionnaire) and 'unchanged' patients (not achieving the MCID), as previously described. ${ }^{18}$

\section{RESULTS}

Out of 338 patients with COPD referred for PR during the study time period, a total of 261 patients completed PR with pre and post CCQ measurements. The non-completers had significantly higher mean CCQ scores than the completers (3.3 vs $2.8 ; \mathrm{p}<0.01)$, despite similar age and lung function parameters. Baseline characteristics of the completers are outlined in table 1.

The baseline CCQ total scores stratified by spirometric GOLD stage were: GOLD $1=2.3$, GOLD $2=2.6$, GOLD $3=2.9$ and GOLD $4=3.2$ (ANOVA, $\mathrm{p}<0.001$ ). The response to $\mathrm{PR}$ is outlined in table 2 .

There were significant changes in ISW, SGRQ, CRQ, CAT and CCQ with PR. The effect sizes of response for SGRQ, CRQ, CAT and CCQ were $-0.33,0.62,-0.25$, and -0.39 , respectively. Change in CCQ with PR correlated significantly with change in SGRQ, CRQ and CAT (table 3; figure 1). 
Table 1 Baseline characteristics: $n=261$. Data expressed as mean (95\% Cls).

\begin{tabular}{|c|c|}
\hline Characteristic & Baseline (pre PR) \\
\hline Age (years) & 71 (70 to 72 ) \\
\hline Sex (F/M) & $110 / 151$ \\
\hline BMI $\left(\mathrm{kg} / \mathrm{m}^{2}\right)$ & 27.6 (26.9 to 28.3$)$ \\
\hline $\mathrm{FEV}_{1}$ (\% predicted) & 49.8 (47.3 to 52.3 ) \\
\hline \multicolumn{2}{|l|}{ Patients (\%) } \\
\hline GOLD 1 & 10 \\
\hline GOLD 2 & 36 \\
\hline GOLD 3 & 32 \\
\hline GOLD 4 & 22 \\
\hline MRC & 3.2 (3.1 to 3.4 ) \\
\hline ISW (m) & 227 (208 to 245$)$ \\
\hline CCQ Total & 2.8 (2.6 to 2.9$)$ \\
\hline GOLD 1 & 2.3 (1.8 to 2.7$)$ \\
\hline GOLD 2 & 2.6 (2.3 to 2.8$)$ \\
\hline GOLD 3 & 2.9 (2.6 to 3.2 ) \\
\hline GOLD 4 & 3.2 (3.0 to 3.5 ) \\
\hline CCQ Symptoms & 1.1 (1.0 to 1.2 ) \\
\hline CCQ Functional & 1.1 (1.1 to 1.2$)$ \\
\hline CCQ Mental & 0.5 (0.5 to 0.6$)$ \\
\hline
\end{tabular}

Change in CCQ with PR, according to baseline GOLD spirometric classification, showed no between-group differences (ANOVA, $\mathrm{p}=0.31$ ).

Floor and ceiling effects were negligible. No patient in the study, either pre or post PR, reached the highest possible score of 6 (worst health status), and only one patient $(<0.01 \%)$ had optimal health status (ie, a score of 0 ).

Table 2 Response to pulmonary rehabilitation (PR): $n=261$. Data expressed as mean $(95 \% \mathrm{Cls})$.

\begin{tabular}{lcc}
\hline Outcome & Change with PR & p Value \\
\hline MRC & $-0.7(-0.8$ to -0.5$)$ & $<0.001$ \\
ISW (m) & $63(55$ to 72$)$ & $<0.001$ \\
SGRQ Total & $-5.3(-6.7$ to -3.4$)$ & $<0.001$ \\
SGRQ Symptoms & $-4.6(-6.4$ to -2.7$)$ & $<0.001$ \\
SGRQ Activities & $-3.7(-5.8$ to -1.7$)$ & $<0.001$ \\
SGRQ Impact & $-6.2(-7.9$ to -4.5$)$ & $<0.001$ \\
CRQ Total & $14.1(11.9$ to 16.4$)$ & $<0.001$ \\
CRQ Dyspnoea & $4.3(3.5$ to 5.1$)$ & $<0.001$ \\
CRQ Fatigue & $3.0(2.5$ to 3.6$)$ & $<0.001$ \\
CRQ Emotion & $3.9(3.0$ to 4.9$)$ & $<0.001$ \\
CRQ Mastery & $2.9(2.2$ to 3.5$)$ & $<0.001$ \\
CAT & $-1.9(-2.7$ to -1.1$)$ & $<0.001$ \\
CCQ Total & $-0.5(-0.6$ to -0.3$)$ & $<0.001$ \\
CCQ Symptoms & $-0.1(-0.2$ to -0.1$)$ & $<0.001$ \\
CCQ Functional & $-0.2(-0.1$ to -0.3$)$ & $<0.001$ \\
CCQ Mental & $-0.1(-0.1$ to -0.1$)$ & $<0.001$
\end{tabular}

CAT, COPD Assessment Test; CCQ, Clinical COPD Questionnaire; CRQ, Chronic Respiratory Questionnaire; ISW, incremental shuttle walk; MRC, Medical Research Council Dyspnoea Score; SGRQ, St George's Respiratory Disease Questionnaire.
Table 3 Univariate correlates of change in CCQ score.

\begin{tabular}{lcc}
\hline Variable & Pearson correlation & p Value \\
\hline$\Delta$ SGRQ Total & 0.48 & $<0.001$ \\
$\Delta$ SGRQ Symptoms & 0.14 & $<0.001$ \\
$\Delta$ SGRQ Activities & 0.38 & $<0.001$ \\
$\Delta$ SGRQ Impact & 0.45 & $<0.001$ \\
$\Delta$ CRQ Total & -0.56 & $<0.001$ \\
$\Delta$ CRQ Dyspnoea & -0.46 & $<0.001$ \\
$\Delta$ CRQ Fatigue & -0.37 & $<0.001$ \\
$\Delta$ CRQ Emotion & -0.44 & $<0.001$ \\
$\Delta$ CRQ Mastery & -0.46 & $<0.001$ \\
$\Delta$ CAT & 0.54 & $<0.001$ \\
\hline$\Delta$ C
\end{tabular}

$\Delta$, delta change; CAT, COPD Assessment Test; CRQ, Chronic Respiratory

Questionnaire; SGRQ, St George's Respiratory Disease Questionnaire.

\section{MCID for the CCQ}

The SEM for the CCQ was 0.29 , half SD change was 0.48 and $\mathrm{MDC}_{95}$ was 0.80 . For patients achieving the MCID for the SGRQ, CRQ and CAT with PR, mean (95\% CI) change in CCQ was $-0.80(-0.96$ to -0.64$),-0.78(-0.92$ to -0.64$)$ and $-0.86(-1.01$ to -0.71 ) (figure 2 ). For those failing to achieve the MCID for the SGRQ, CRQ and CAT with PR, mean (95\% CI) change in CCQ was $-0.13(-0.28$ to 0.02$),-0.01(-0.17$ to 0.16$)$ and $0.00(-0.14$ to 0.15$)$ (figure 2 ).

ROC curves to identify the best change in CCQ to discriminate between those achieving the MCID for the SGRQ, CRQ and CAT are shown in figure 3. All three curves were consistent in identifying a CCQ change cutoff of -0.40 as the best discriminating value with an area under the curve of $0.71,0.75$ and 0.77 , respectively. Using the proposed MCID of -0.40 , $54 \%$ of the PR cohort achieved the MCID for the CCQ. In comparison, 52\%, 58\% and 53\% achieved the established MCID for the SGRQ, CRQ and CAT.

\section{DISCUSSION}

The main findings of the present study are that the CCQ is responsive to outpatient $\mathrm{PR}$ and is equally as responsive as other well established HRQoL questionnaires such as the SGRQ and CAT. Change in CCQ in response to PR correlates significantly with change in SGRQ, CRQ and CAT. Furthermore, using three different HRQoL questionnaires as external anchors, we were able to obtain a consistent estimate of the minimal clinically important improvement for the CCQ to be -0.4 , corroborating previous estimates.

\section{CCQ and PR}

Despite the CCQ being first described and validated in $2003,{ }^{5}$ it is surprising that there is a paucity of data regarding the response of the CCQ to PR. Damato et al demonstrated a significant reduction in total CCQ score (from 2.0 to 1.3 ) in 46 patients with COPD undergoing 3 weeks of intensive inpatient PR. ${ }^{19}$ More recently, Dodd et al examined the response of the CAT to outpatient PR in a prospective multicentre centre. ${ }^{8}$ In one of the seven participating centres, the CCQ was also measured before and after PR in 57 patients, demonstrating a significant mean reduction of -0.7 . Strengths of our current study include the significantly larger sample size, the concurrent prospective data collection of several HRQoL questionnaires in all patients, and the unselected nature of patients with COPD who were referred to PR for clinical reasons. 

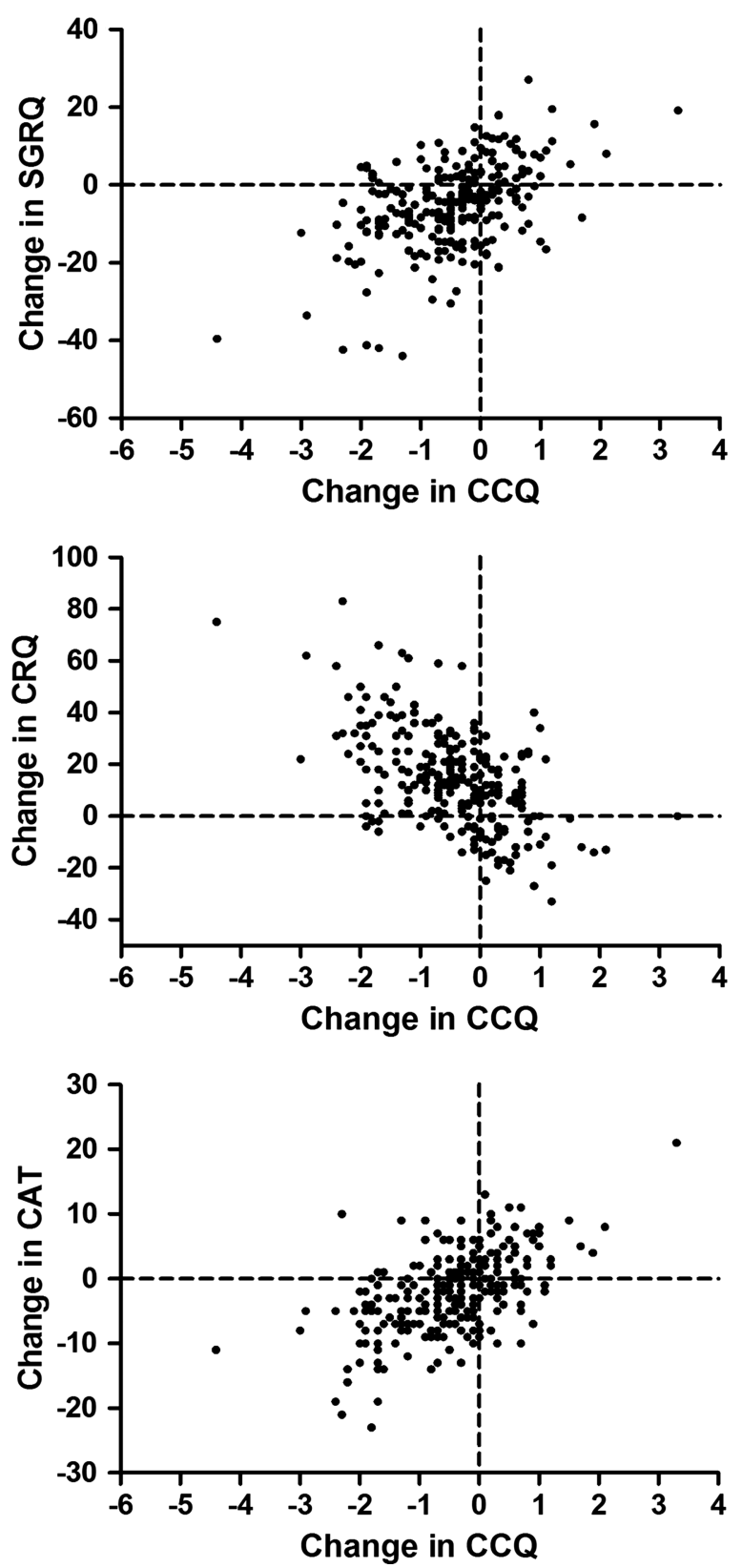

Figure 1 Change in Clinical COPD Questionnaire (CCQ) in response to pulmonary rehabilitation correlated with change in St George's Respiratory Questionnaire (SGRQ), Chronic Respiratory Questionnaire (CRQ) and COPD Assessment Test (CAT) (Pearson correlation $r=0.48$, -0.56 and 0.54 , respectively; all $p<0.001)$.

Several recent studies have proposed the CAT as a simpler practical alternative HRQoL measure to more time-consuming questionnaires such as the CRQ and SGRQ, ${ }^{8} 1020$ arguing that the adoption of a quicker health status questionnaire could lead to more efficient service delivery and consequent cost savings. Like the CAT, the CCQ requires considerably less time and staff input to complete compared with more established questionnaires, ${ }^{6}$ but the CCQ may arguably provide greater information due to the inclusion of domain and total scores. Recent studies also seem to suggest that at the patient and clinician level, there is a slight preference for the CCQ over the CAT. ${ }^{679}$ According to effect size, the CCQ appeared to be as responsive, if not more responsive, than the CAT and the SGRQ, corroborating previous data, ${ }^{8}$ albeit from a smaller cohort.

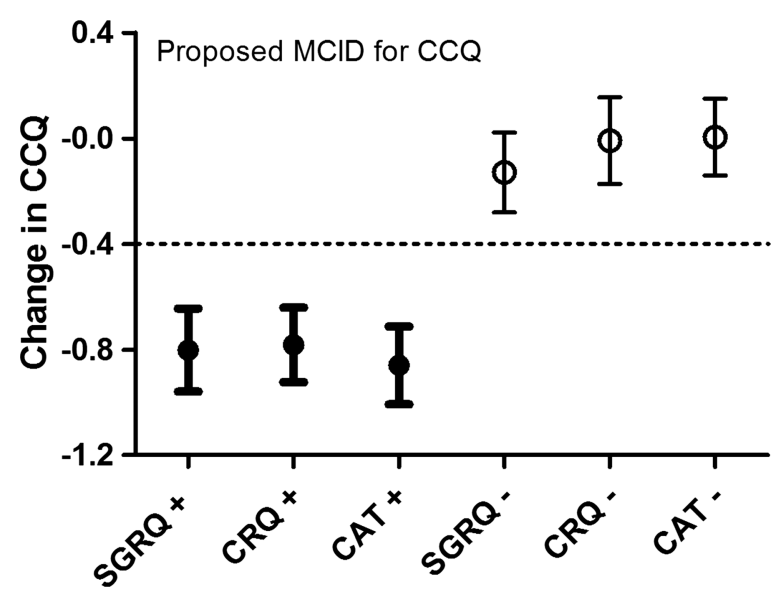

Figure 2 Mean $(95 \% \mathrm{Cl})$ change in Clinical COPD Questionnaire (CCQ) with pulmonary rehabilitation, according to achievement (+) or non-achievement (-) of the established minimal clinically important difference (MCID) for the St George's Respiratory Questionnaire (SGRQ), Chronic Respiratory Questionnaire (CRQ) and COPD Assessment Test (CAT). The dotted line represents the proposed MCID of 0.4 .

The minimal clinically important difference for the CCQ Although the CCQ is responsive to intervention and change, 5192122 there is a paucity of data regarding the MCID. One previous study calculated the SEM only ${ }^{7}$ and only one study has attempted to estimate the MCID of the CCQ using more than one approach. ${ }^{12}$ Kocks et al studied 210 hospitalised patients with acute exacerbations of COPD participating in a randomised controlled trial comparing 5 days of treatment with either intravenous or oral prednisolone. Using a distributionbased approach, the SEM was calculated to be $0.21 .^{12}$ The investigators also used the global rating of change questionnaire as an external criterion to anchor the change in CCQ. On day 3 after admission the mean (95\% CI) change in CCQ in 22 patients reporting a pre-specified global rating of +2 to +3 was $-0.44(-0.13$ to -0.75$)$. In 168 patients who completed a CCQ at day 42 after admission, differences in mean CCQ scores at day 42 were compared between patients who did and did not experience a major health event (death, rehospitalisation) at 12 months. A mean difference of 0.39 was observed, again with wide $95 \%$ CIs (0.07 to 0.71$)^{12}$

We believe that our current study adds to the current knowledge base as there were significant differences to the study of Kocks et al. First, our study focused on stable patients with COPD rather than those admitted with an acute exacerbation. Second, we used changes in HRQoL questionnaires as our external anchors, which measure similar construct to the CCQ. This was confirmed by demonstrating that change in CCQ with PR correlated significantly with change in the other HRQoL questionnaires with coefficients ranging from 0.48 to 0.56 . This is important as robust anchor-based estimates are dependent on significant correlations between the outcome of interest and the anchors. ${ }^{23}$ Lastly, we used ROC curves to plot sensitivity/specificity plots to identify the CCQ change cutoff that best identified patients who achieved the MCID for the anchor questionnaires. Despite using three different anchors, it was extremely reassuring to observe the same estimate for the MCID of the CCQ. We believe that using the mean of the 'improvers' overestimates the MCID as the group may include those who experience a minimal clinically important improvement and those who experience large clinical important. To illustrate the case, the SGRQ 'improvers' had a mean SGRQ change of -13.3 

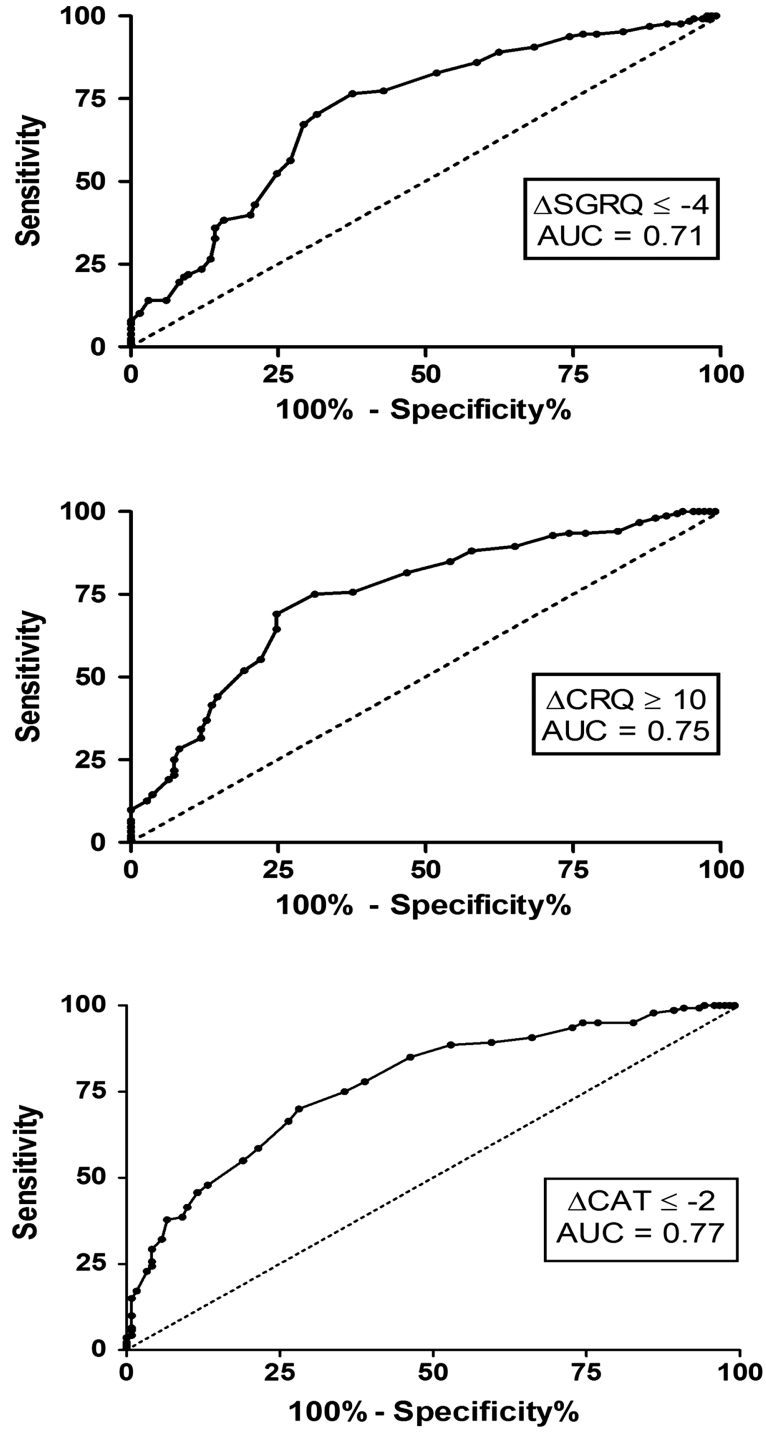

Figure 3 Receiver operating characteristics curves demonstrating the sensitivity and specificity of change in Clinical COPD Questionnaire (CCQ) in predicting patients who improve by at least the minimal clinically important difference for the St George's Respiratory Questionnaire (SGRQ), Chronic Respiratory Questionnaire (CRQ) and COPD Assessment Test (CAT). For all measures, a CCQ change of -0.4 was identified as the best cutoff. The dotted line represents the line of identity. AUC, area under the curve.

following PR, noticeably greater than the accepted MCID for the SGRQ. Despite these differences, our findings corroborate the findings of Kocks and colleagues in estimating the MCID for the CCQ to be 0.4 .

There were limitations to our study. Only a few patients showed deterioration in the HRQoL greater than the accepted MCID for the questionnaires. Due to the small numbers, we were not able to anchor the change in CCQ against significant deterioration in HRQoL. Hence our estimate of the MCID is strictly only an estimate of the minimal clinically important improvement. Further studies in the CCQ are required to confirm that patients perceive size of deterioration similarly to size of improvement. Recall bias is another universal limitation of all approaches that use changes in questionnaires over time as the external anchor. In addition, subjective responses to external anchors will be highly biased by the patient's explicit knowledge of the change in outcome of interest. A further limitation is that patients who did not complete PR were excluded from the final analysis. Although the PR completion rate of $77 \%$ is in line with, if not better than, rates described from other UK services, it is conceivable that the exclusion of the non-completers may have biased estimates of responsiveness of the CCQ.

In summary, our study demonstrates that the CCQ is responsive to the effects of PR with similar, if not better, responsiveness to the CAT and SGRQ. Using a variety of HRQoL questionnaires as external anchors, we estimate the minimal clinically improvement of the CCQ to be a 0.4 point decrease. We propose that the CCQ is a practical, simpler alternative to more time-consuming measures of HRQoL.

Acknowledgements The authors would like to acknowledge the Harefield Pulmonary Rehabilitation Unit at Harefield Hospital for their assistance in collecting the data.

Contributors Recruitment of patients, collection of data: all authors except MIP. Analysis of data: all authors. Preparation of manuscript: all authors. WD-CM conceived the idea and is the guarantor of the paper, taking responsibility for the integrity of the work as a whole, from inception to published article. SSCK and DD contributed equally.

Funding SSCK is supported by the Medical Research Council (MRC). WD-CM is supported by a National Institute for Health Research Clinician Scientist Award (CS/ 7/007), a Medical Research Council (UK) New Investigator Research Grant (G1002113) and a National Institute for Health Research Clinical Trials Fellowship (NIHR-CTF-01-12-04). This project was undertaken at the NIHR Respiratory Biomedical Research Unit at the Royal Brompton and Harefield NHS Foundation Trust and Imperial College London; JLC, SEJ and MIP's salaries are wholly or part funded by the Biomedical Research Unit. The views expressed in this publication are those of the authors and not necessarily those of the NHS, The National Institute for Health Research or the Department of Health.

Competing interests None.

Ethics approval National Research Ethics Committee.

Provenance and peer review Not commissioned; externally peer reviewed.

\section{REFERENCES}

1 Vestbo J, Hurd SS, Agusti AG, et al. Global strategy for the diagnosis, management, and prevention of chronic obstructive pulmonary disease: GOLD executive summary. Am J Respir Crit Care Med 2013:187:347-65.

2 Jones PW, Quirk FH, Baveystock CM. The St George's Respiratory Questionnaire. Respir Med 1991;85(Suppl B):25-31; discussion 3-7.

3 Williams JE, Singh SJ, Sewell L, et al. Development of a self-reported Chronic Respiratory Questionnaire (CRQ-SR). Thorax 2001;56:954-9.

4 Jones PW, Harding G, Berry P, et al. Development and first validation of the COPD Assessment Test. Eur Respir J 2009;34:648-54.

5 van der Molen T, Willemse BW, Schokker S, et al. Development, validity and responsiveness of the Clinical COPD Questionnaire. Health Qual Life Outcomes 2003;1:13.

6 Ringbaek T, Martinez G, Lange P. A comparison of the assessment of quality of life with CAT, CCQ, and SGRQ in COPD patients participating in pulmonary rehabilitation. COPD 2012;9:12-5.

7 Tsiligianni IG, van der Molen T, Moraitaki D, et al. Assessing health status in COPD. A head-to-head comparison between the COPD Assessment Test (CAT) and the Clinical COPD Questionnaire (CCQ). BMC Pulm Med 2012;12:20.

8 Dodd JW, Hogg L, Nolan J, et al. The COPD Assessment Test (CAT): response to pulmonary rehabilitation. A multicentre, prospective study. Thorax 2011; 66:425-9.

9 Cave AJ, Atkinson L, Tsiligianni IG, et al. Assessment of COPD wellness tools for use in primary care: an IPCRG initiative. Int I Chron Obstruct Pulmon Dis 2012;7:447-56

10 Dodd JW, Marns PL, Clark AL, et al. The COPD Assessment Test (CAT): short- and medium-term response to pulmonary rehabilitation. COPD 2012;9:390-4.

11 Jones PW, Harding G, Wiklund I, et al. Tests of the responsiveness of the Chronic Obstructive Pulmonary Disease (COPD) Assessment Test TM (CAT) following acute exacerbation and pulmonary rehabilitation. Chest 2012;142:134-40.

12 Kocks JW, Tuinenga MG, Uil SM, et al. Health status measurement in COPD: the minimal clinically important difference of the clinical COPD questionnaire. Respir Res 2006;7:62.

13 Pauwels RA, Buist AS, Calverley PM, et al. Global strategy for the diagnosis, management, and prevention of chronic obstructive pulmonary disease. NHLBI/WHO 
Global Initiative for Chronic Obstructive Lung Disease (GOLD) Workshop summary. Am J Respir Crit Care Med 2001;163:1256-76.

14 Bolton CE, Bevan-Smith EF, Blakey JD, et al. British Thoracic Society guideline on pulmonary rehabilitation in adults: accredited by NICE. Thorax 2013;68(Suppl 2): ii1-30.

15 Singh SJ, Morgan MD, Scott S, et al. Development of a shuttle walking test of disability in patients with chronic airways obstruction. Thorax 1992;47:1019-24.

16 Jones PW. St. George's Respiratory Questionnaire: MCID. COPD 2005;2:75-9.

17 Schunemann HJ, Puhan M, Goldstein R, et al. Measurement properties and interpretability of the Chronic Respiratory Disease Questionnaire (CRQ). COPD 2005;2:81-9.

18 Jones SE, Kon SS, Canavan JL, et al. The five-repetition sit-to-stand test as a functional outcome measure in COPD. Thorax 2013. Published Online First 19 June 2013 doi: 10.1136/thoraxjnl-2013-203576
19 Damato S, Bonatti C, Frigo V, et al. Validation of the Clinical COPD Questionnaire in Italian language. Health Qual Life Outcomes 2005;3:9.

20 Kon SS, Clark AL, Dilaver D, et al. Response of the COPD Assessment Test to pulmonary rehabilitation in unselected chronic respiratory disease. Respirology 2013;18:974-7.

21 Trappenburg JC, Monninkhof EM, Bourbeau J, et al. Effect of an action plan with ongoing support by a case manager on exacerbation-related outcome in patients with COPD: a multicentre randomised controlled trial. Thorax 2011;66:977-84.

22 Tashkin DP, Rennard S, Taylor Hays J, et al. Lung function and respiratory symptoms in a 1-year randomized smoking cessation trial of varenicline in COPD patients. Respir Med 2011;105:1682-90.

23 Redelmeier DA, Bayoumi AM, Goldstein RS, et al. Interpreting small differences in functional status: the Six Minute Walk test in chronic lung disease patients. Am J Respir Crit Care Med 1997;155:1278-82.

Applications are invited for the post of

\section{Editor, Thorax}

Thorax is a leading international peer-reviewed journal in respiratory medicine. The BMJ and British Thoracic Society seek an editor who can advance the journal's scientific reputation while maintaining its relevance to practicing respiratory physicians worldwide.

Applications are invited from specialists in any branch of respiratory medicine. Joint applications will be considered. Applicants need not be based in the UK.

Full editorial support and training will be provided. The editor should expect to spend up to a day in total each week on journal-related activities. The successful applicant(s) will be free to select his/her own team of associate editors and board members.

Closing date for applications is Tuesday 30 September 2014. Interviews will be held in early November 2014 at BMJ offices in London, UK. It is envisaged that the successful candidate will officially take up the post of Editor in July 2015, following a hand over period with the current editors. Term of office is usually 5 years.

Further details of the post can be discussed with the Journals Manager, Claire Weinberg, and/or the retiring editors, Andrew Bush and Ian Pavord. A job description is available on request from Mrs Weinberg (cweinberg@bmj.com).

Applications should be addressed to Ms Kelly Horwood, BMJ, BMA House, Tavistock Square, London WC1H 9JR or email khorwood@bmj.com 